\title{
Erratum
}

\section{Characterization of a genome-specific Gypsy-like retrotransposon sequence and development of a molecular marker specific for Dasypyrum villosum (L.)}

Jie Zhang, Hai Long, Zhifen Pan, Junjun Liang, Shuiyang Yu, Guangbing Deng and Maoqun Yu

J. Genet. 92, 103-108

The caption for figure 2 appears under figure3, and the caption for figure 3 appears under figure 2. The captions should be interchanged. Figures 2 and 3 should also be in reverse order, i.e. the sequence alignment figure (printed and in online file as figure 3 ) should be figure 2, and the FISH figure should be figure 3. 